\title{
Characterization of deltamethrin degradation and metabolic pathway by co-culture of Acinetobacter junii LH-1-1 and Klebsiella pneumoniae BPBA052
}

\author{
Jie Tang ${ }^{*}$ (D, Qiong Hu, Dan Lei, Min Wu, Chaoyi Zeng and Qing Zhang
}

\begin{abstract}
Deltamethrin and its major metabolite 3-phenoxybenzoic acid (3-PBA) have caused serious threat to the environment as well as human health, yet little is known about their degradation pathways by bacterial co-cultures. In this study, the growth and degradation kinetics of Acinetobacter junii LH-1-1 and Klebsiella pneumoniae BPBA052 during deltamethrin and 3-PBA degradation were established, respectively. When the inoculum proportion of the strains LH-1-1 and BPBA052 was 7.5:2.5, and LH-1-1 was inoculated $24 \mathrm{~h}$ before inoculation of strain BPBA052, 94.25\% deltamethrin was degraded and $9.16 \mathrm{mg} / \mathrm{L}$ of 3-PBA remained within $72 \mathrm{~h}$, which was $20.36 \%$ higher and $10.25 \mathrm{mg} / \mathrm{L}$ lesser than that in monoculture of LH-1-1, respectively. And the half-life of deltamethrin was shortened from $38.40 \mathrm{~h}$ to $24.58 \mathrm{~h}$. Based on gas chromatography-mass spectrometry, 3-phenoxybenzaldehyde, 1,2-benzenedicarboxylic butyl dacyl ester, and phenol were identified as metabolites during deltamethrin degradation in co-culture. This is the first time that a co-culture degradation pathway of deltamethrin has been proposed based on these identified metabolites. Bioremediation of deltamethrin-contaminated soils with co-culture of strains LH-1-1 and BPBA052 significantly enhanced deltamethrin degradation and 3-PBA removal. This study provides a platform for further studies on deltamethrin and 3-PBA biodegradation mechanism in co-culture, and it also proposes a promising approach for efficient bioremediation of environment contaminated by pyrethroid pesticides and their associated metabolites.
\end{abstract}

Keywords: Deltamethrin, 3-phenoxybenzoic acid, Co-culture, Acinetobacter junii LH-1-1, Klebsiella pneumoniae BPBA052

\section{Keypoints}

- Co-culture was used to degrade both deltamethrin and its toxic intermediate metabolite 3-PBA.

- Co-culture was more efficient in degradation of deltamethrin and 3-PBA than the monoculture.

- A degradation pathway in co-degradation of deltamethrin and 3-PBA was first reported.

\footnotetext{
*Correspondence: wendyjiejie@tom.com
}

Key Laboratory of Food Biotechnology, School of Food

and Biotechnology, Xihua University, Chengdu 610039, Sichuan, People's

Republic of China
- Deltamethrin-contaminated soils could be efficiently bioremediated by co-culture.

\section{Introduction}

Pyrethroid pesticides (PPs) are a type of synthetic insecticides, which are widely used in agriculture, forestry, horticulture, public health, and indoor places to control pests, cockroaches, mosquitoes, and livestock parasites because of their broad spectrum, high efficiency, and low mammalian toxicity as compared to that of organophosphate pesticides (Cycoń and Piotrowska-Seget 2016; Zhan et al. 2018). Deltamethrin, belongs to type II PPs, which 
has high insecticidal activity and has been commonly and widely used against insect pests (Hao et al. 2018). However, its continuous and extensive using not only resulted in serious contamination of the environment, but also negatively affected the non-target organisms ( $\mathrm{Li}$ et al. 2008), as it shows neurotoxicity, reproductive toxicity, immunotoxicity, and acts as an endocrine disruptor (Zhang et al. 2010; Brander et al. 2016). Recently, deltamethrin has received increasing attention because of its major metabolite, 3-phenoxybenzoic acid (3-PBA) (Hao et al. 2018; Braganca et al. 2019; Zhang et al. 2016). Many studies have suggested that 3-PBA with stronger hydrophilicity is more likely to spread in the environment than its parent compounds, making it difficult to degrade and easy to accumulate (Chen et al. 2012b). Moreover, 3-PBA is a toxic and persistent hydrophobic compound, and its antibacterial activity is a major obstacle for microbial growth and further degradation of its parent compound (Chen et al. 2011b, 2012b; Hu et al. 2018). Meanwhile, it is considered as an endocrine disruptor due to its antiestrogenic activity (Chen et al. 2011b, 2012a). Therefore, effective measures for simultaneous degrading and removal of deltamethrin and its intermediate metabolite 3-PBA are crucial to ensure that human and environmental health is not influenced by the excessive use of deltamethrin.

Currently, biodegradation has become a mainstream technology for removal of pesticide residues or pesticide metabolites, as it is eco-friendly, cost-effective, convenient, and possesses excellent degradation properties (Hao et al. 2018). At present, a variety of microorganisms have been used to degrade pesticides or their metabolites, such as Bacillus sp. (Liu et al. 2015; Chen et al. 2015; Tang et al. 2018b), Pseudomonas sp. (Halden et al. 1999), Sphingomonas sp. (Tang et al. 2013), Stenotrophomonas sp. (Chen et al. 2011c), and Aspergillus sp. (Zhu et al. 2016; Deng et al. 2015). However, most of these microorganisms cannot completely mineralize pesticides or their metabolites alone (Chen et al. 2012b). Zhao et al. (2016) reported that about $50 \% \beta$-cypermethrin $(100 \mathrm{mg} / \mathrm{L})$ could be degraded by Bacillus licheniformis B-1 after $72 \mathrm{~h}$, but was unable to sustain mineralization of 3-PBA; Zhu et al. (2016) reported that $80.62 \%$ 3-PBA (100 mg/L) could be degraded by Aspergillus oryzae M-4 within $5 \mathrm{~d}$, but did not clear its ability to degrade PPs. Therefore, a new idea emerged, wherein synergy between strains to simultaneously degrade pyrethroid pesticides and their toxic intermediate metabolites (Jones and Wang 2018). Compared with other biodegradation methods, in cocultures, microbes are more adaptable to the environment and can achieve more rapid pathway optimization, thereby improving the efficiency of pollutant degradation (Zhao et al. 2016; Jones and Wang 2018). Recently, some reports have verified that microbial co-cultures can effectively degrade organic pollutants and their related metabolites (Zhao et al. 2016; Tran et al. 2013). Liu et al. (2014) studied the co-degradation of cypermethrin and 3-PBA by strains Bacillus licheniformis B-1 and Sphingomonas sp. SC-1, the degradation rate of cypermethrin $(100 \mathrm{mg} / \mathrm{L})$ was $75.60 \%$ in $72 \mathrm{~h}$ and the content of 3-PBA was $10.31 \mathrm{mg} / \mathrm{L}$. As compared with the degradation by strain B- 1 alone, the degradation of cypermethrin increased by $24.52 \%$ and $3-\mathrm{PBA}$ content reduced by $12.5 \mathrm{mg} / \mathrm{L}$ in case of degradation of cypermethrin in coculture. However, co-culture pathways for degradation of deltamethrin and its intermediate metabolites have remained largely unexplored.

In this study, co-culture of Acinetobacter junii LH-1-1 and Klebsiella pneumoniae BPBA052 was used for simultaneous degradation of deltamethrin and its toxic intermediate metabolite 3-PBA. The growth and degradation kinetics, inoculum ratio, inoculation sequence, and soil bioremediation ability of the co-culture strains were studied. In addition, this report contains the first proposal of a microbial co-culture degradation pathway for degrading deltamethrin and 3-PBA based on metabolite identification. This study also provided a promising approach for further bioremediation environment contaminated by PPs or 3-PBA.

\section{Materials and methods}

\section{Chemicals and culture conditions}

Deltamethrin (98.5\%) was purchased from Ronch Chemicals (Nanjing, China), 3-PBA standard (98.0\%) and chromatographic-grade acetonitrile were obtained from TCI (Shanghai, China) and Adamas-Beta (Shanghai, China), respectively. Other chemicals were analytical grade and commercially available.

The liquid medium used for degradation of deltamethrin by the bacteria was optimized mineral salt medium (OMSM), containing $0.5 \mathrm{~g} / \mathrm{L} \mathrm{KH}_{2} \mathrm{PO}_{4}, 1.5 \mathrm{~g} / \mathrm{L} \mathrm{K}_{2} \mathrm{HPO}_{4}$, $1.5 \mathrm{~g} / \mathrm{L}\left(\mathrm{NH}_{4}\right)_{2} \mathrm{SO}_{4}, 0.2 \mathrm{~g} / \mathrm{L} \mathrm{MgSO}_{4}, 0.5 \mathrm{~g} / \mathrm{L} \mathrm{NaCl}, 0.01 \mathrm{~g} / \mathrm{L}$ $\mathrm{FeCl}_{3}$, and supplemented with $3 \mathrm{~g} / \mathrm{L}$ tryptone as extra carbon source, the $\mathrm{pH}$ was adjusted to 7.0. At each stage, pre-cultures were incubated in Luria-Bertani (Tang et al. 2018a) (LB) liquid medium for $24 \mathrm{~h}$ at $30^{\circ} \mathrm{C}$, harvested by centrifugation at $8000 \mathrm{rpm}$ for $2 \mathrm{~min}$, washed twice with $0.9 \%$ sterile $\mathrm{N}$-saline, and adjusted to a cell density about $1.0 \times 10^{8}$ cells $/ \mathrm{mL}$ to prepare the inoculum. All the experiments were performed in triplicate, and non-inoculated cultures served as controls.

\section{Microorganisms}

A. junii LH-1-1 (Collection number: CGMCC 7.378, GenBank accession number: MK053916) was isolated from strawberry rhizosphere soil and could degrade 
$82.36 \%$ of deltamethrin $(75 \mathrm{mg} / \mathrm{L})$ at $30{ }^{\circ} \mathrm{C}$ in OMSM after incubation for 96 h. Klebsiella pneumoniae BPBA052 (Collection number: CGMCC 7.307, GenBank accession number: KY425746) was obtained from the soybean rhizosphere soil, and it could degrade $96.37 \%$ of 3-PBA (100 mg/L) within $72 \mathrm{~h}$ in mineral salt medium (Tang et al. 2019).

\section{Growth and degradation kinetics of degrading strains}

Considering that a growth and degradation kinetics model can predict the status of degrading-strain and residue of pollutants in the future (Kamyabi et al. 2018; Kuppusamy et al. 2016), a logistic equation (Eq. 1) was used to describe the kinetics of bacterial growth, and a first-order-degradation kinetic model (Eq. 2) was used to describe the residue of substrates (deltamethrin or 3-PBA), where $X_{0}$ represents the initial cell concentration $\left(\mathrm{OD}_{600}\right), X_{m}$ represents the maximum cell concentration $\left(\mathrm{OD}_{600}\right), t$ represents the culture time (h), and $\mu_{m}$ represents the maximum specific growth rate $\left(\mathrm{h}^{-1}\right) . C_{0}$ is the initial substrates concentration $(\mathrm{mg} / \mathrm{L}), C_{t}$ is the substrates concentration at time $t(\mathrm{mg} / \mathrm{L}), t$ is the degradation time (h), $k$ is the degradation rate constant $\left(\mathrm{h}^{-1}\right)$, and $t_{1 / 2}$ is the half-life of substrates. Inoculum $(5.0 \% \mathrm{v} / \mathrm{v})$ was added to $100 \mathrm{~mL}$ OMSM containing $75 \mathrm{mg} / \mathrm{L}$ substrates in a $250 \mathrm{~mL}$ Erlenmeyer flask, $\mathrm{OD}_{600}$ and substrates concentration were measured at regular intervals.

$$
\begin{aligned}
& X=X_{0} e^{u_{m} t} /\left(1-\left(X_{0} / X_{m}\right)\left(1-e^{u_{m} t}\right)\right) \\
& C_{t}=C_{0} \times e^{-k t}, t_{1 / 2}=\ln 2 / k
\end{aligned}
$$

\section{Effect of inoculum proportion of strains LH-1-1 and BPBA052 on deltamethrin and 3-PBA degradation}

The strains LH-1-1 and BPBA052 were incubated in LB liquid medium to prepare inoculum. With a total inoculum of $5 \%(\mathrm{v} / \mathrm{v})$, strains LH-1-1 and BPBA052 were mixed at a proportion of 10:0, 7.5:2.5, 6.7:3.3, 5:5, 3.3:6.7, 2.5:7.5, and 0:10. Each inoculum was inoculated to $100 \mathrm{~mL}$ OMSM with $75 \mathrm{mg} / \mathrm{L}$ deltamethrin for $72 \mathrm{~h}$ to measure the residues of deltamethrin and 3-PBA, and non-inoculated cultures served as controls.

\section{Effect of inoculation sequences of strains LH-1-1 and BPBA052 on deltamethrin and 3-PBA degradation} Under the optimal inoculum proportion of strains LH-1-1 and BPBA052, three different inoculation sequences of strains LH-1-1 and BPBA052 were investigated. Sequence 1: LH-1-1 was first inoculated and cultured in OMSM for $24 \mathrm{~h}$ before inoculation of strain BPBA052. Sequence 2: strains LH-1-1 and BPBA052 were inoculated in the OMSM, simultaneously.
Sequence 3: BPBA052 was first inoculated and cultured in OMSM for 24 h before inoculation of strain LH-1-1. Each sequence was inoculated to $100 \mathrm{~mL}$ OMSM with $75 \mathrm{mg} / \mathrm{L}$ deltamethrin at an interval of $12 \mathrm{~h}$ to measure the residues of deltamethrin and 3-PBA, and non-inoculated cultures served as controls.

\section{Metabolite characterization}

Three sets of experiments (L, LP, C) were set up to investigate the degradation of deltamethrin and its metabolic products in the co-culture. In experiment $\mathrm{L}$, $5 \%(\mathrm{v} / \mathrm{v})$ inoculum of strain $\mathrm{LH}-1-1$ was inoculated in $200 \mathrm{~mL}$ OMSM containing $75 \mathrm{mg} / \mathrm{L}$ of deltamethrin as a positive control. In experiment LP, under the optimal inoculation proportion and inoculation sequence, coculture of strains LH-1-1 and BPBA052 (total inoculum of $5 \%(\mathrm{v} / \mathrm{v}))$ were inoculated in OMSM. In experiment $\mathrm{C}$, non-inoculated cultures served as negative control. The metabolites of deltamethrin at an interval of $12 \mathrm{~h}$ were extracted and identified by gas chromatographymass spectrometry (GC-MS) as described by Tang et al. (2018a).

\section{Degradation of deltamethrin and its intermediate metabolite 3-PBA in soil environment}

Soil samples were collected from top $0-10 \mathrm{~cm}$ of a field in Xihua University, Sichuan, China, which had never been contaminated by pyrethroid insecticides. The characteristics of the collected soil samples were as follows: total bacteria: $4.7 \times 10^{8} \mathrm{CFU} / \mathrm{g}, \mathrm{pH}: 6.38$, organic matter: $18.40 \mathrm{~g} / \mathrm{kg}$, total nitrogen: $2.52 \mathrm{~g} / \mathrm{kg}$, total phosphorus: $0.43 \mathrm{~g} / \mathrm{kg}$, water content: $34.52 \%(\mathrm{w} / \mathrm{w})$. After the soil was air-dried and sieved, deltamethrin was added to the soil to make the final concentration $20 \mathrm{mg} / \mathrm{kg}$, and then kept overnight for further studies.

Both sterile and nonsterile soils were used to investigate the co-culture effect of strains LH-1-1 and BPBA052 on degradation of deltamethrin and its metabolite, 3-PBA. Each soil sample (L, LP, C) was prepared by using $500 \mathrm{~g}$ of nonsterile soil in $500 \mathrm{~mL}$ Erlenmeyer flask. In sample L, only strain LH-1-1 was added in the nonsterile soil. In sample LP, under the optimal inoculation proportion and inoculation sequence, co-culture of strains LH-1-1 and BPBA052 was added in the nonsterile soil. And non-inoculated, nonsterile soil served as sample $C$ (control). The mentioned above experiments (L, LP, C) were also performed once again in sterile soil. All the experiments were performed in triplicate. The water content of these soil samples was maintained at $35.0 \pm 5.0 \%$ and the temperature was maintained at $30 \pm 5{ }^{\circ} \mathrm{C}$. The 
soil samples were collected and the content of deltamethrin and 3-PBA was measured every day.

\section{Results}

Growth and degradation kinetics of the bacterial strains

As shown in Fig. 1, the experimental values of the biomass $\left(\mathrm{OD}_{600}\right)$ of strains were nonlinearly fitted in the growth kinetics model. The $\mathrm{OD}_{600}$ of strain LH-1-1 rose from 0.06 to 0.74 and the growth kinetics parameters were $\mu_{\mathrm{m}}=0.12346 \mathrm{~h}^{-1}, X_{0}=0.06183, X_{\mathrm{m}}=0.74619$ and substituting (Eq. 1) for the growth kinetics equation of strain LH-1-1: $X_{\mathrm{LH}-1-1}=0.06183 \mathrm{e}^{0.12346 t} /(1-0.082861(1-$ $\left.\left.\mathrm{e}^{0.12346 \mathrm{t}}\right)\right), \mathrm{R}^{2}=0.986$. The results of growth kinetics of BPBA052 in OMSM containing $75 \mathrm{mg} / \mathrm{L}$ of 3-PBA were as follows: $X_{\mathrm{BPBA} 052}=0.0918 \mathrm{e}^{0.09419 t} /(1-0.080152(1-$ $\left.\left.\mathrm{e}^{0.09419 \mathrm{t}}\right)\right)$, where $\mu_{\mathrm{m}}=0.09419 \mathrm{~h}^{-1}, \quad X_{0}=0.0918$, $X_{\mathrm{m}}=1.14532, \mathrm{R}^{2}=0.985$.

The first-order degradation kinetics model (Eq. 2) was used to nonlinearly fit the substrate residues in the degradation process. Within $96 \mathrm{~h}$, more than $82 \%$ of the $75 \mathrm{mg} / \mathrm{L}$ deltamethrin initially added to the medium was degraded by LH-1-1 and a first-order degradation kinetics equation was obtained: $C_{\mathrm{LH}-1-1}=74.02463 \mathrm{e}^{-0.01805 t}$, $k=0.01805, t_{1 / 2}=38.40 \mathrm{~h}, \mathrm{R}^{2}=0.996$. The formation of 3-PBA was accompanied with the degradation of deltamethrin, reaching a maximum concentration of $22.40 \mathrm{mg} / \mathrm{L}$ in $96 \mathrm{~h}$. It was noteworthy that the $\mathrm{OD}_{600}$ of strain LH-1-1 was 0.74, which was far lower than that of the strain BPBA052 (1.04). It may be because the toxic intermediate 3-PBA has antibacterial activity and inhibits microbial growth (Chen et al. 2011a). The first-order degradation kinetics equation of strain BPBA052 to degrade 3-PBA was as follows: $C_{\mathrm{BPBA} 052}=75.04785 \mathrm{e}^{-0.03063 t}$, $k=0.03063, t_{1 / 2}=22.63 \mathrm{~h}, \mathrm{R}^{2}=0.995$, and it showed
93.34\% of 3-PBA $(75 \mathrm{mg} / \mathrm{L})$ was degraded within $96 \mathrm{~h}$ in OMSM. The degradation rate was slightly slower in OMSM than in mineral salt medium, this inhibitory effect may be related to metabolism of additional carbon sources, which was also observed in the case of strain ZS-19 (Chen et al. 2015).

The $\mathrm{R}^{2}$ of the growth and degradation kinetics of strain LH-1-1 were 0.986 and 0.996 , respectively. For the strain BPBA052 the values were 0.985 and 0.995 , respectively. These results indicated that the two models can be quite well reflect the actual growth regularity of strains and the degradation regularity of substrates (deltamethrin or 3-PBA) by LH-1-1 or BPBA052, respectively.

\section{Effect of inoculum proportion on the degradation of deltamethrin and its metabolite 3-PBA}

Based on the degradation of different substrates by $A$. junii LH-1-1 and $K$. pneumoniae BPBA052, the coculture of these two strains was used to improve the degradation of deltamethrin and decrease the content of metabolite 3-PBA during deltamethrin degradation process. As shown in Fig. 2, with the decrease in the amount of $A$. junii LH-1-1 inoculum and the increase in the amount of inoculum of $K$. pneumoniae BPBA052, the degradation rate of deltamethrin first increased and then decreased, while the content of 3-PBA gradually decreased within $72 \mathrm{~h}$. When only strain LH-1-1 (10:0) was inoculated, the degradation rate of deltamethrin was $73.90 \%$ and a large amount of 3-PBA was accumulated (up to $19.41 \mathrm{mg} / \mathrm{L}$ ), suggesting that the strain LH-1-1 could not effectively degrade 3-PBA, which could have also inhibited further degradation of deltamethrin. When only strain BPBA052 (0:10) was inoculated, the residue of 3-PBA was the least, but the degradation rate of
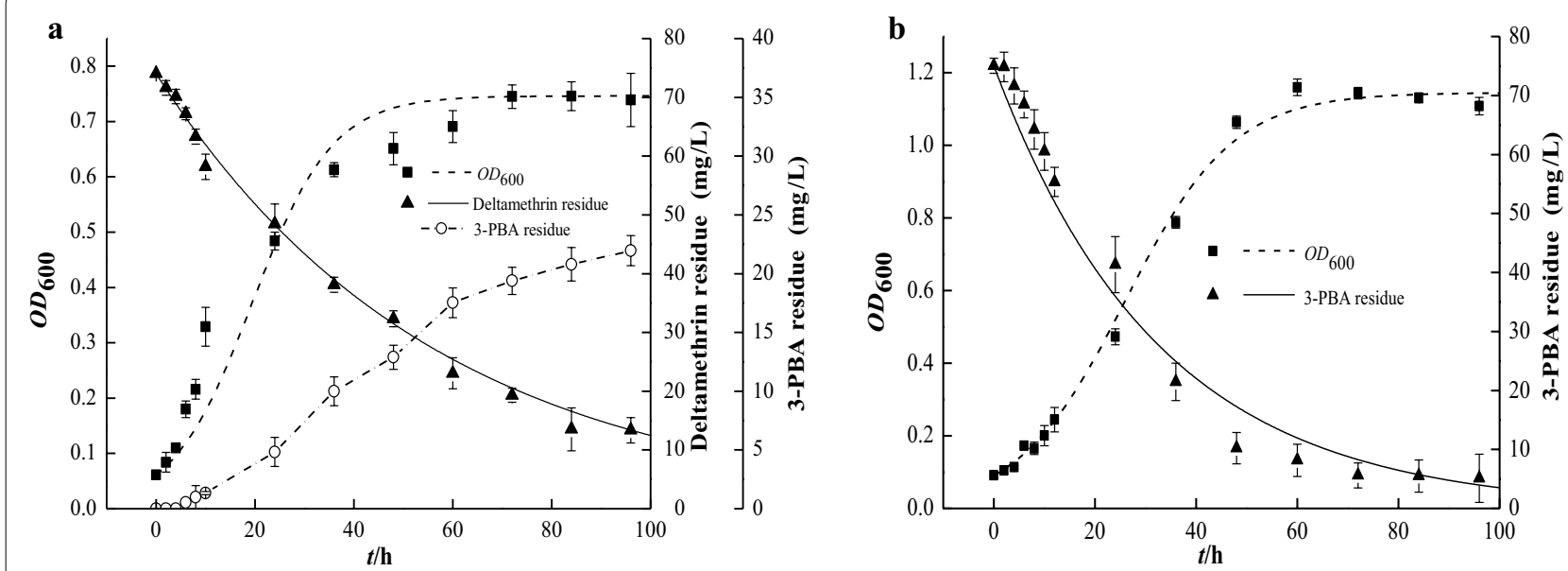

Fig. 1 Growth and degradation curve of A. junii LH-1-1 a and K. pneumoniae BPBA052 b 


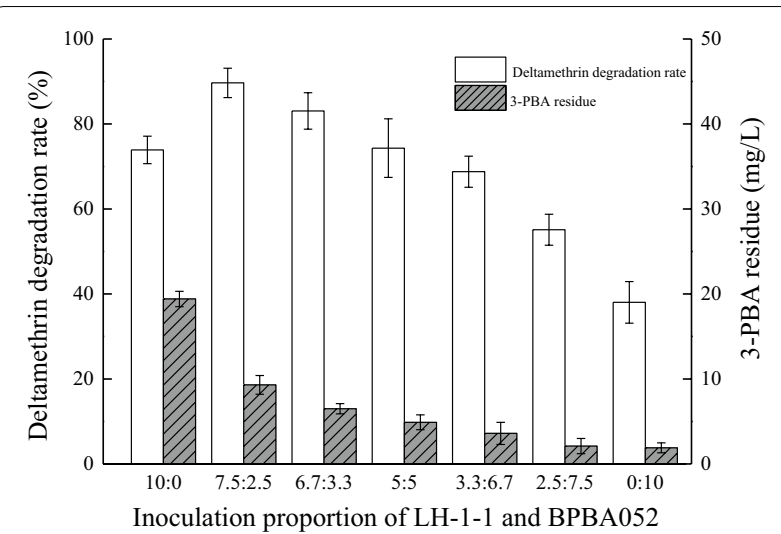

Fig. 2 Deltamethrin degradation and 3-PBA content by using different inoculation proportions of strains $\mathrm{LH}-1-1$ and BPBA052

deltamethrin was only $38.03 \%$, because BPBA052 is an efficient 3-PBA degrading strain with poor deltamethrindegrading ability. However, when the inoculation proportion of LH-1-1 and BPBA052 was 7.5:2.5, the degradation rate of deltamethrin reached the highest- $89.67 \%$, and only $9.30 \mathrm{mg} / \mathrm{L} 3-\mathrm{PBA}$ remained at $72 \mathrm{~h}$. Therefore, in our study the most appropriate proportion of strains LH-1-1: BPBA052 was 7.5:2.5, which used for subsequent studies.

\section{Effect of inoculation sequences on the degradation of deltamethrin and its metabolite 3-PBA}

Based on the optimal inoculum proportion of the strains LH-1-1 and BPBA052, the optimal inoculation sequence of the strains for preparation of the co-culture was investigated. As shown in Fig. 3a, b, the residue of 3-PBA was not significantly different among the three different inoculation sequences within $72 \mathrm{~h}$, but it is worth noting that there was a continuous degradation trend of 3-PBA, suggesting that 3-PBA might be completely metabolized with prolonged degradation time. But the degradation rate of deltamethrin by using sequences 1,2 , and 3 were $94.25 \%, 90.23 \%$, and $81.45 \%$ within $72 \mathrm{~h}$, respectively. This indicated that sequence 1 was the most efficient method, and the first-order degradation kinetics equation of the two strains by using sequence 1 to degrade deltamethrin was obtained: $C_{\mathrm{LH}-1-1+\mathrm{BPBA} 052}=75.411 \mathrm{e}^{-0.0282 t}$, $k=0.0282, t_{1 / 2}=24.58 \mathrm{~h}, \mathrm{R}^{2}=0.974$. The degradation rate of deltamethrin was $20.35 \%$ higher than that in monoculture A. junii LH-1-1, and the half-life was shortened from $38.40 \mathrm{~h}$ to $24.58 \mathrm{~h}$. The results showed that deltamethrin could be efficiently co-degraded by $A$. junii LH-1-1 and K. pneumoniae BPBA052.

\section{Metabolic products and degradation pathway of deltamethrin by co-culture}

To reveal the degradation mechanism of deltamethrin by the co-culture of strains $A$. junii LH-1-1 and K. pneumoniae BPBA052, samples were detected and characterized by GC-MS (Fig. 4). And three main degradation products (Fig. 5 and Table 1) were identified during the degradation process with those of corresponding authentic standard compounds from the National Institute of Standards and Technology (NIST) library database.

In experiment L (monoculture LH-1-1), three compounds were detected (Fig. $4 \mathrm{a}$ ) within $72 \mathrm{~h}$, compound A with significant peaks at retention times of 39.27 and $41.68 \mathrm{~min}$ showed major fragment ions at $\mathrm{m} / \mathrm{z} 181$ and 253 , which was identified as deltamethrin. With the disappearance of compound A, two new compounds B and $\mathrm{C}$ were formed. According to their retention times and
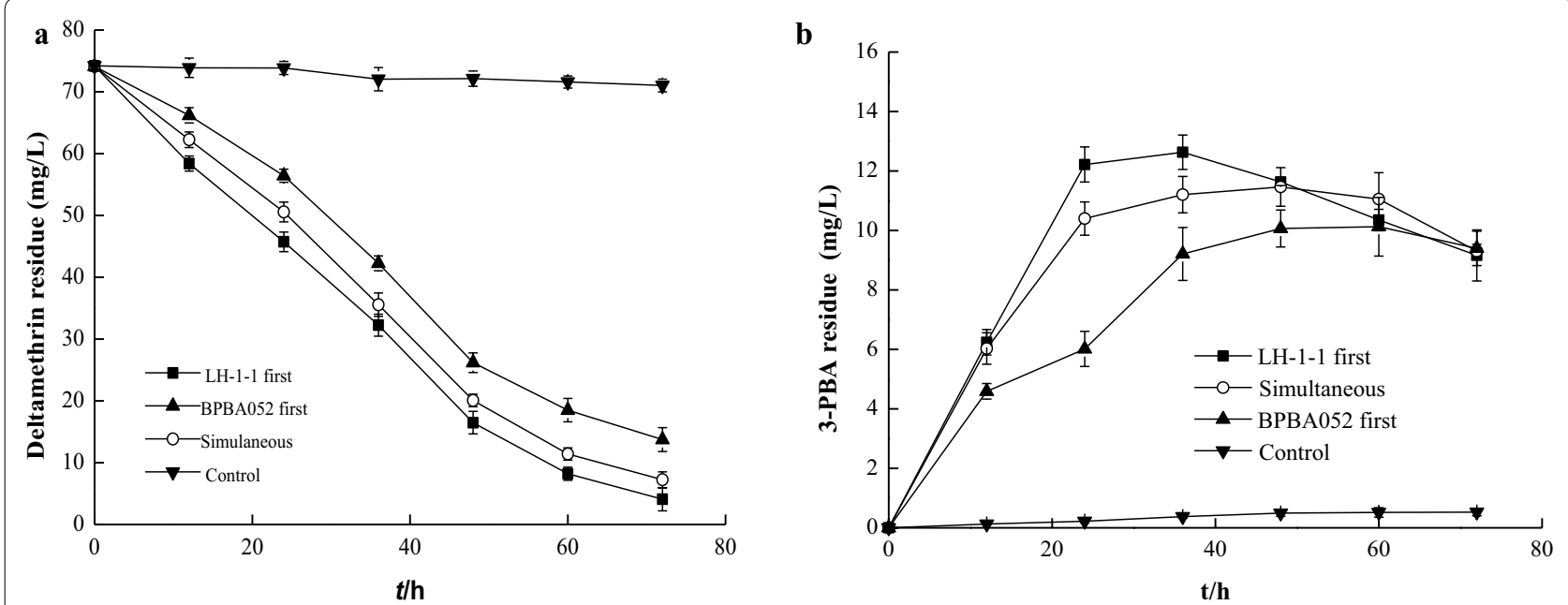

Fig. 3 Effect of inoculation sequences of strains A. junii LH-1-1 and K. pneumoniae BPBA052 on degradation of deltamethrin a and its metabolite 3-PBA (b) 
$(\mathbf{x} 1,000,000)$

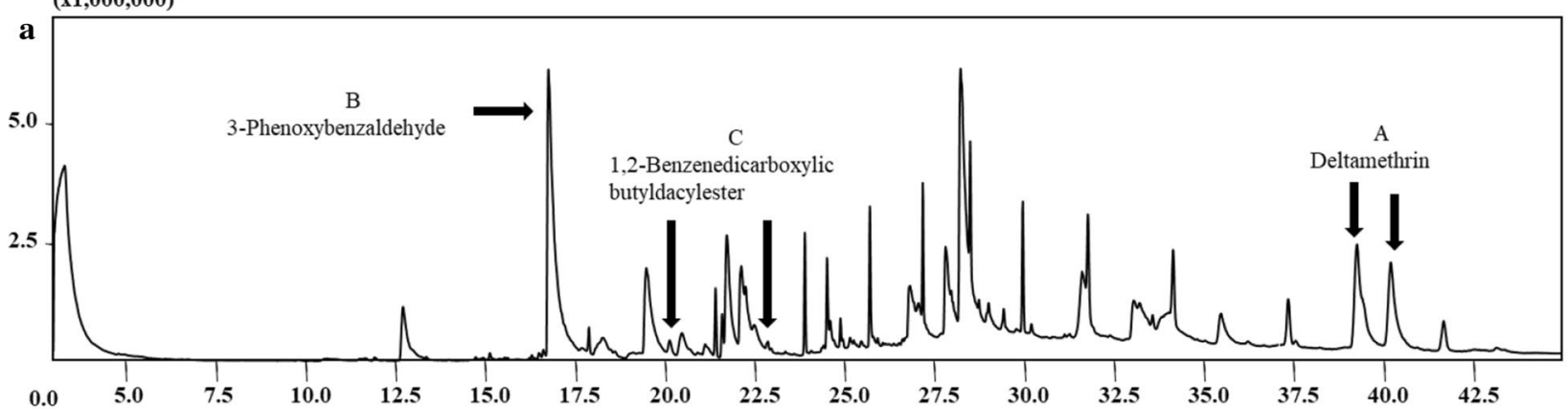

$(\mathbf{x} 1,000,000)$

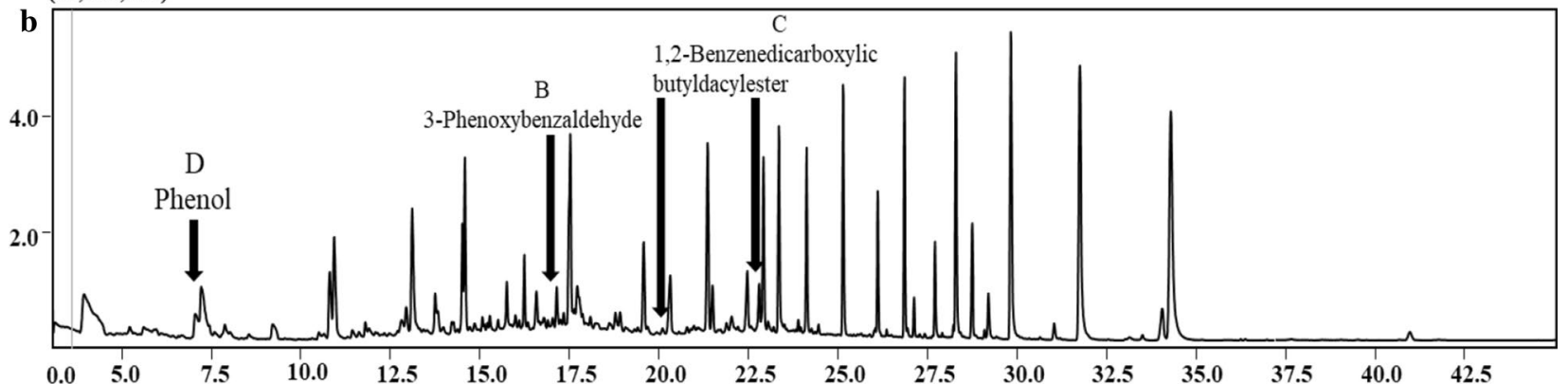

Fig. 4 a GC-MS chromatogram of metabolites of deltamethrin obtained by degradation of deltamethrin in monoculture strain $A$. junii LH-1-1 within $72 \mathrm{~h}$; and $\mathbf{b}$ co-culture strains A. junii LH-1-1 and K. pneumoniae BPBA052 within $72 \mathrm{~h}$

molecular ions after matching with the NIST library database, compound B was characterized as intermediate 3-phenoxybenzaldehyde, which was unstable and easily dehydrogenated into 3-PBA according to the research of $\mathrm{Hu}$ et al. (2018). Compound C was 1,2-benzenedicarboxylic butyl dacyl ester. It is noteworthy that, for the first time, compound $\mathrm{C}$ has been detected in the biodegradation pathway of deltamethrin.

In experiment LP, the intermediate metabolites from co-culture degradation of deltamethrin were 3-phenoxybenzaldehyde (B), 1,2-benzenedicarboxylic butyl dacyl ester (C), and phenol (D) within $72 \mathrm{~h}$. A new peak with retention time 6.525 min was observed and was identified as phenol, which was not detected in experiment $\mathrm{L}$ (monoculture strain LH-1-1). The formation of phenol may be due to the oxidization and cleavage of diaryl ether of 3-PBA by the strain BPBA052 (Tang et al. 2019). Along with metabolization, no persistent accumulative product was detected, the results showed that deltamethrin and its intermediate metabolites were degraded and completely mineralized by co-culture. Based on the chemical structures of deltamethrin and the identified metabolites, a microbial metabolic pathway of deltamethrin by co-culture of strains $A$. junii LH-1-1 and K. pneumoniae BPBA052 was proposed (Fig. 6). Firstly, deltamethrin (A) was hydrolyzed to yield 3-phenoxybenzaldehyde (B) and
(1R, cis)-3-(2,2-dibromoethenyl)-2,2-dimethylcyclopropane carboxylic acid by strain LH-1-1. Secondly, a small amount of toxic intermediate 3-phenoxybenzaldehyde (B) was further metabolized with diaryl cleavage to form 1,2-benzenedicarboxylic butyl dacyl ester by strain LH-11 , and through the cleavage of aromatic rings to achieve complete metabolism. While a large amount of transient 3-phenoxybenzaldehyde (B) was dehydrogenated into 3-PBA, which was rapidly degraded with oxidation and cleavage of diaryl ether to generate phenol (D) by strain BPBA052. Finally, phenol was converted to $\mathrm{CO}_{2}$ and $\mathrm{H}_{2} \mathrm{O}$ by the strain BPBA052 to achieve complete mineralization (Tang et al. 2019).

\section{Degradation of deltamethrin and its intermediate metabolite 3-PBA in soil environment}

The content of deltamethrin and 3-PBA in the sterile soil and nonsterile soils after degradation by monoculture strain $A$. junii LH-1-1 (L sample), co-culture strains $A$. junii LH-1-1 and $K$. pneumoniae BPBA052 (LP sample), and the control (C sample) are shown in Fig. 7. LP sample in nonsterile soil after incubation $7 \mathrm{~d}$, showed $77.33 \%$ degradation of the $20 \mathrm{mg} / \mathrm{kg}$ deltamethrin initially added to the soil, and 3-PBA was not detected. While in L sample, the degradation of deltamethrin was only $68.10 \%$ and $3.878 \mathrm{mg} / \mathrm{kg}$ of $3-\mathrm{PBA}$ was detected. These results 


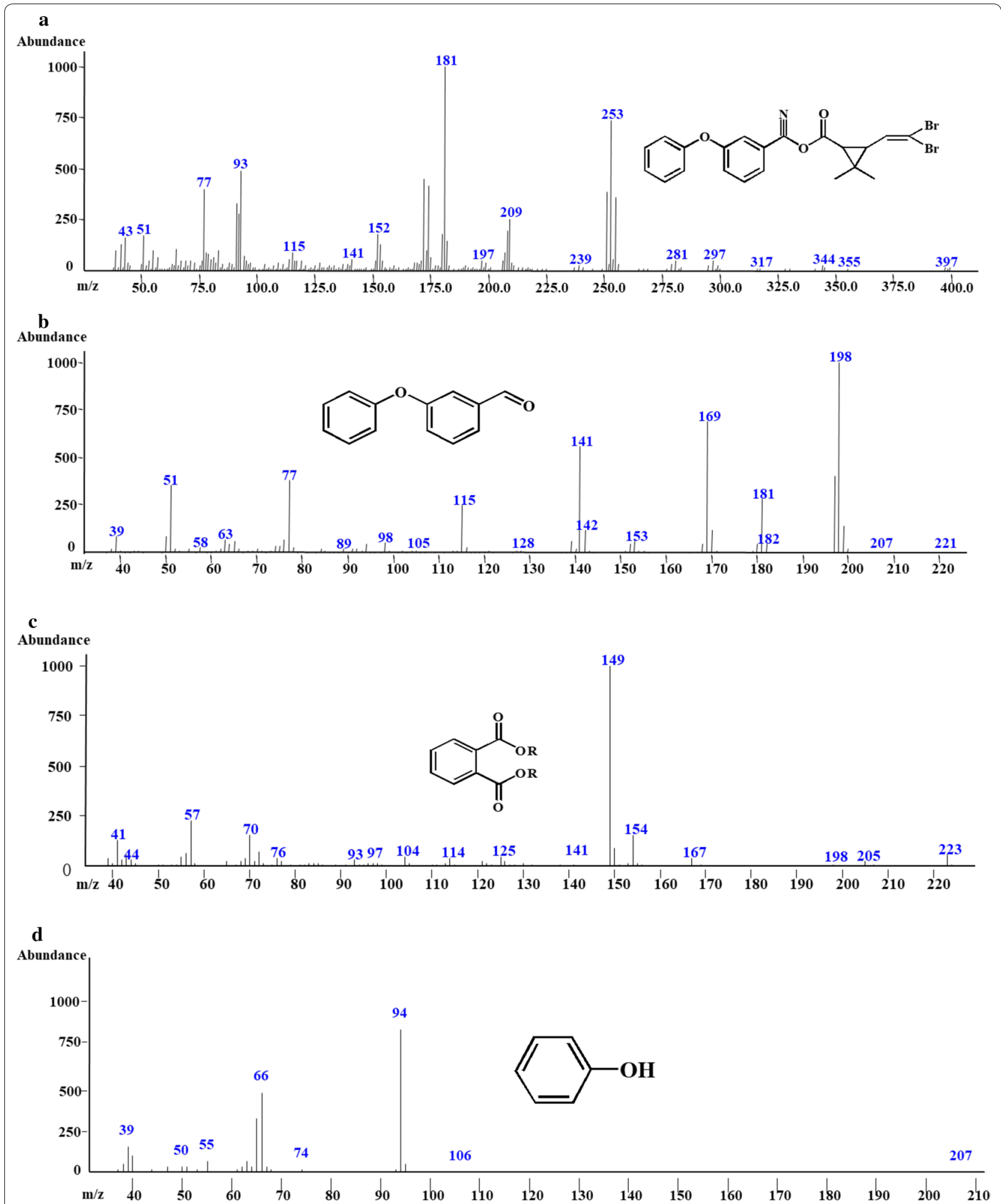

Fig. 5 a-c $m / z$ of compounds (A-C) in GC-MS from deltamethrin degradation by strain LH-1-1. b-d m/z of compounds (B-D) in GC-MS from deltamethrin degradation by co-culture of strains $\mathrm{LH}-1-1$ and BPBA052 
Table 1 Chromatographic properties of metabolites of deltamethrin by strain LH-1-1, co-culture strains LH-1-1 and BPBA052

\begin{tabular}{|c|c|c|c|c|c|}
\hline Compound & $\mathrm{m} / \mathrm{z}$ & Retention time (min) & $\begin{array}{l}\text { Chemical structural formula } \\
\text { in NIST library }\end{array}$ & Similarity (\%) & Name \\
\hline A & 505 & $39.267 / 41.683$ & & 93 & Deltamethrin \\
\hline B & 198 & 16.792 & & 95 & 3-Phenoxybenzaldehyde \\
\hline$C$ & 278 & $20.167 / 22.275$ & & 96 & $\begin{array}{l}\text { 1,2-benzenedicarboxylic } \\
\text { butyl dacyl ester }\end{array}$ \\
\hline D & 94 & 6.525 & & 95 & Phenol \\
\hline
\end{tabular}

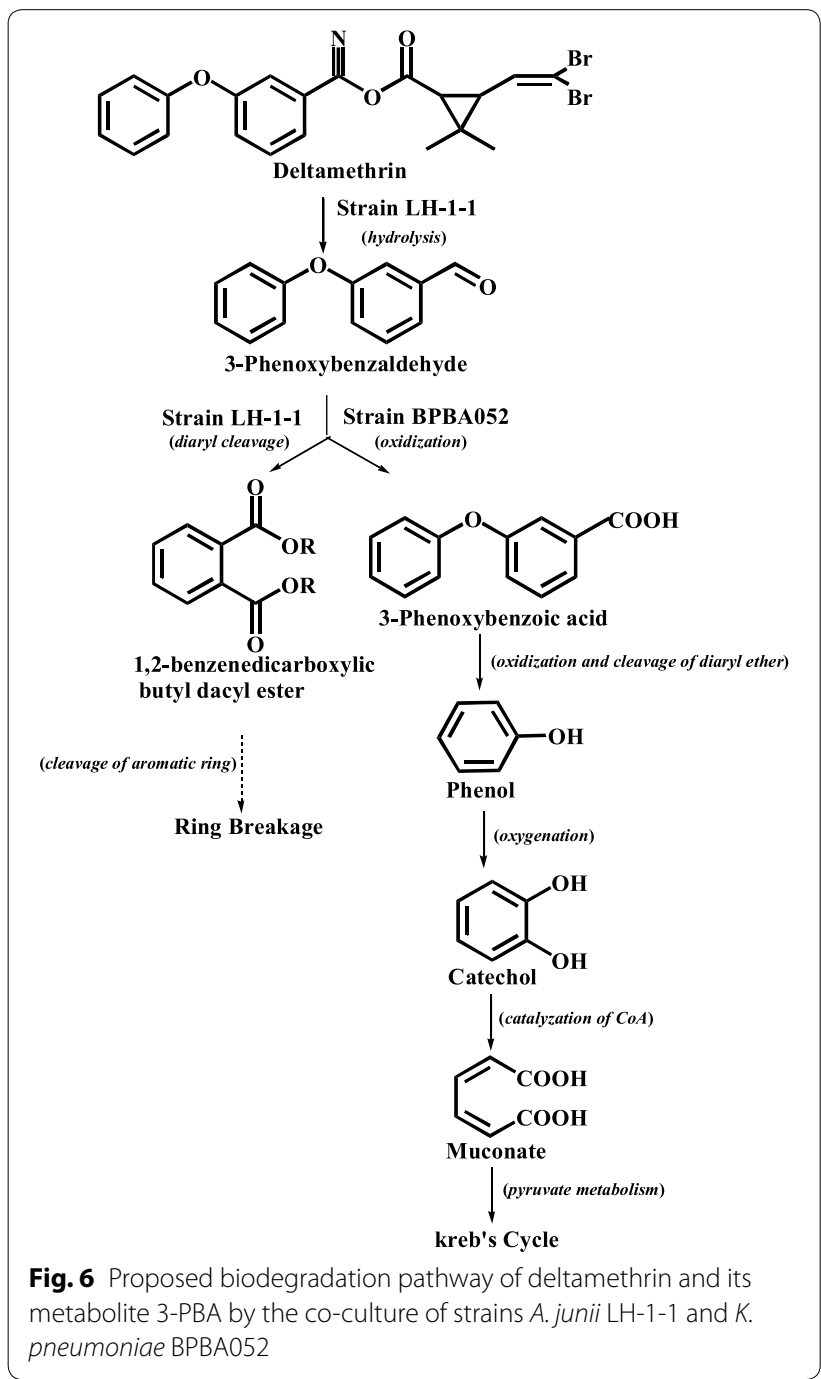

showed that both in the medium or the natural soil environment, bioremediation by co-culture of strains LH-1-1 and BPBA052 was better than that in monoculture strain LH-1-1, as described previously by Liu et al. (2014). Similar results were also found in sterile soil, the degradation rate of deltamethrin in LP sample was $72.65 \%$, which was $10.63 \%$ higher than that in L sample. And the content of 3-PBA was $0.18 \mathrm{mg} / \mathrm{kg}$ in LP sample, which was $4.26 \mathrm{mg} /$ $\mathrm{kg}$ lower than that in L sample. These results further confirmed that co-culture strains LH-1-1 and BPBA052 has promising potential and advantages as bioremediation organisms in degrading deltamethrin and its intermediate metabolite 3-PBA in highly various environments.

\section{Discussion}

Up to now, most studies effort has focused on screening and isolating highly effective pesticide-degrading strains from pesticide-contaminated environments, while ignoring how to improve the degradation of pesticides and the completely mineralized pathway of pesticides and their associated metabolites (Chen et al. 2011c; Deng et al. 2015). Here, when the inoculum proportion of the strains LH-1-1 and BPBA052 was 7.5:2.5, and LH-1-1 was inoculated $24 \mathrm{~h}$ before inoculation of strain BPBA052, the degradation of deltamethrin and 3-PBA concentration by co-culture of strains LH-1-1 and BPBA052 was 20.36\% higher and $10.25 \mathrm{mg} / \mathrm{L}$ lesser than that in monoculture LH-1-1. This may be due to that strain LH-1-1 cannot quickly and completely degrade toxic intermediate 3-PBA, while BPBA052 possess high-efficient degradation ability in 3-PBA. Therefore, when a proper amount of BPBA052 was introduced, the toxicity inhibition of 3-PBA was quickly released in the degradation process, and the degradation of deltamethrin was further accelerated (Zhao et al. 2016). Liu et al. (2014) reported that the appropriate proportion of Bacillus licheniformis B-1 and Sphingomonas sp. SC-1 to degrade cypermethrin 

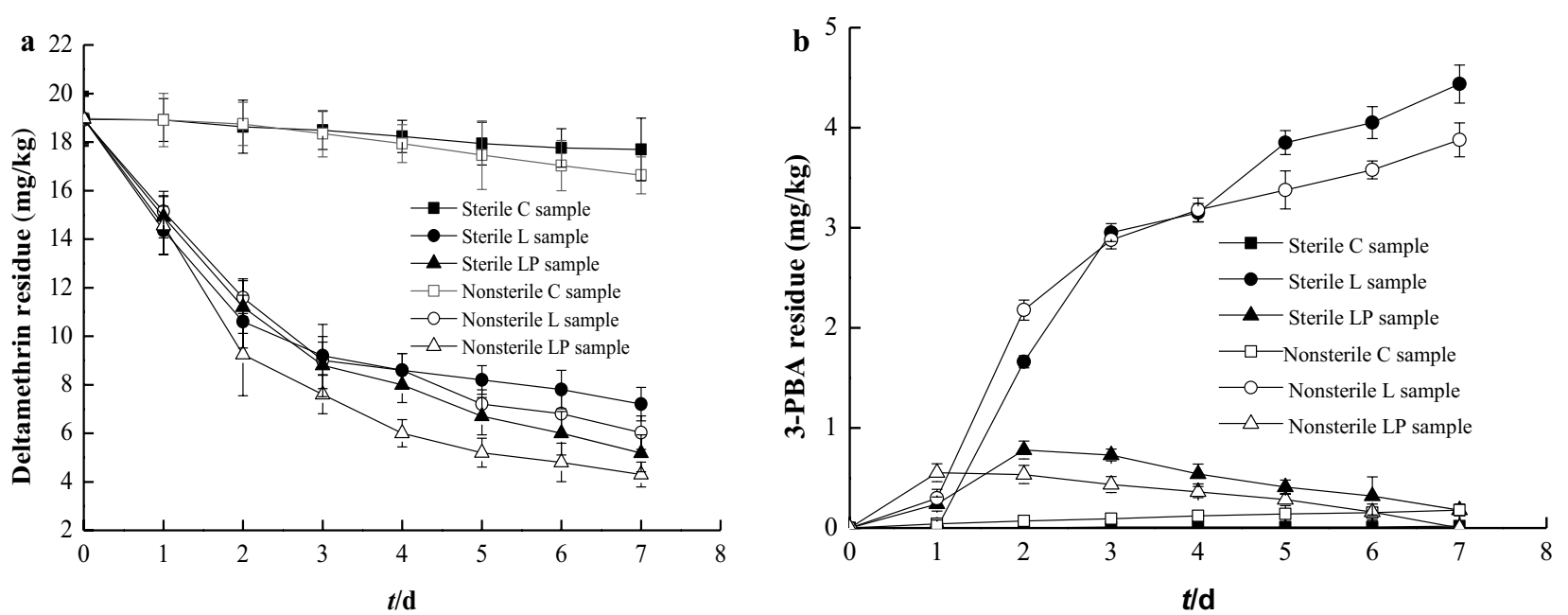

Fig. 7 Deltamethrin residue a and 3-PBA residue $\mathbf{b}$ in the sterile soil and nonsterile soils by monoculture strain A. junii LH-1-1 (L sample), the co-culture A. junii LH-1-1 and K. pneumoniae BPBA052 (LP sample), and the uninoculated control (C sample)

and its metabolite was 3.3:6.7, this signifies that the optimal inoculum proportion in case of different strains and substrates in a co-culture is different. But the inoculation sequence was confirmed the observations made by Liu et al.(2014), pesticide-degrading strains inoculated $24 \mathrm{~h}$ before inoculation of 3-PBA-degrading strains (sequence 1) was the most efficient method. And the degradation rate of deltamethrin and the residue of 3-PBA in our study were higher and lower, respectively, than that in co-culture strains B-1 and SC-1 (Liu et al. 2014). Thus, co-culture strains LH-1-1 and BPBA052 possess broad application potential for further bioremediation of PPs contaminated environment.

Although metabolic mechanism is the basis of application, reports on co-culture biodegradation pathway and mechanism are still rare (Chen et al. 2012b; Zhao et al. 2016). This research verified the degradation mechanism of deltamethrin and 3-PBA by co-culture of strains LH-1-1 and BPBA052 (Fig. 6). Firstly, deltamethrin (A) was hydrolyzed to yield 3-phenoxybenzaldehyde (B) and (1R, cis)-3-(2,2-dibromoethenyl)-2,2-dimethylcyclopropane carboxylic acid by strain LH-1-1. Previous evidences also indicated that esters are considered susceptible to degradation by microbes via hydrolysis, which is the main step in detoxification of PPs (Hu et al. 2018; Yang et al. 2018). This hydrolysis is similar to the initial step of $\beta$-cypermethrin degradation by Bacillus licheniformis B-1 reported by Zhao et al. (2016), wherein $\beta$-cypermethrin was hydrolyzed to 3-PBA. Regrettably, the further degradation of 3-PBA was not observed using strain B-1. Although Halden et al. (1999) and Topp and Akhtar (1991) reported that Pseudomonas sp. strains could utilize and transform 3-PBA, and Chen et al. (2012a) reported that Bacillus sp. DG-02 was capable of oxidizing 3-PBA to form 3-(2-hydroxyphenoxy) benzoic acid. But all these 3-PBA-degrading strains could not metabolize PPs. Fortunately, in our current study coculture of strains LH-1-1 and BPBA052 not only achieved the detoxification of deltamethrin by ester bond hydrolysis, but further transformed its toxic metabolite (3-PBA) by cleavage of the diaryl bond, achieving completely mineralized pathway. That is a small amount of hydrolyzed 3-phenoxybenzaldehyde (B) was metabolized with diaryl cleavage to form 1,2-benzenedicarboxylic butyl dacyl ester by strain LH-1-1, and through the cleavage of aromatic rings to achieve complete metabolism. While a large amount of transient 3-phenoxybenzaldehyde (B) was rapidly degraded with oxidation and cleavage of diaryl ether by BPBA052 to generate phenol releasing the toxicity inhibition of 3-PBA and achieving completely mineralized pathway.

The potential of the co-culture strains LH-1-1 and BPBA052 to remediate deltamethrin- contaminated soil has been confirmed. Whether in sterilized soils or in unsterilized soils, the degradation rate of deltamethrin and 3-PBA concentration in co-cultured strains LH-1-1 and BPBA052 is much higher and lower than that in monoculture strain LH-1-1. And both in case of degradation by monoculture of strain LH-1-1 or co-culture of strains LH-1-1 and BPBA052, the degradation rate of deltamethrin in nonsterile soil was higher than that in sterile soil, and the content of 3-PBA in nonsterile soil was lower than that in sterile soil. These results confirmed the observations made by Chen et al. (2012a) and Zhan et al. 
(2018), suggesting that introduced strains and autochthonous microorganisms may have a synergistic effect in degrading xenobiotic compounds. Therefore, co-culture could be a promising strategy for remediation of environments contaminated with PPs.

In summary, the inoculum proportion and the inoculation sequence played an important role in effective degradation of deltamethrin in co-culture of strains LH-1-1 and BPBA052. And the co-culture degraded deltamethrin via a novel metabolic pathway with the formation of 3-phenoxybenzaldehyde by ester hydrolysis reaction by strain LH-1-1, which was further transformed by strain LH-1-1 and BPBA052 to form 1,2-benzenedicarboxylic butyl dacyl ester and phenol without any persistent accumulative product, indicating that the co-culture harbors a complete metabolic pathway for degradation and mineralization of deltamethrin. Simultaneously, the study revealed that use of co-culture is a potential and efficient strategy for bioremediation of environments contaminated with pyrethroid and their toxic intermediate metabolites.

\section{Acknowledgements}

Not applicable.

\section{Authors' contributions}

JT and $\mathrm{QH}$ designed research and wrote the manuscript; $\mathrm{QH}, \mathrm{DL}$ and $\mathrm{MW}$ conducted experiments; CYZ and QZ analyzed data. All authors read and approved the final manuscript.

\section{Funding}

This work was supported by the Application Foundation Project of Sichuan Provincial Department of Science and Technology (2019YJ0389), the Technological Innovation Project of Chengdu Science and Technology Bureau (2018-YF05-00522-SN), the Key Scientific Research Fund of Xihua University (Z1310525), the Graduate Student Innovation Fund of Xihua University (ycjj2019122).

\section{Availability of data and materials}

The corresponding author is responsible for providing all experimental data upon request.

Ethics approval and consent to participate

Not applicable.

\section{Consent for publication}

All authors give their consent for publication.

\section{Competing interests}

All authors declare that they have no competing interests.

Received: 20 May 2020 Accepted: 27 May 2020

Published online: 03 June 2020

\section{References}

Braganca I, Lemos PC, Delerue-Matos C, Domingues VF (2019) Pyrethroid pesticide metabolite, 3-PBA, in soils: method development and application to real agricultural soils. Environ Sci Pollut Res Intl 26(3):2987-2997

Brander SM, Gabler MK, Fowler NL, Connon RE, Schlenk D (2016) Pyrethroid pesticides as endocrine disruptors: molecular mechanisms in vertebrates with a focus on fishes. Environ Sci Technol 50(17):8977-8992
Chen SH, Hu MY, Liu JJ, Zhong GH, Yang L, Rizwan-ul-Haq M, Han HT (2011a) Biodegradation of beta-cypermethrin and 3-phenoxybenzoic acid by a novel Ochrobactrum lupini DG-S-01. J Hazard Mater 187(1-3):433-440

Chen SH, Lai KP, Li YA, Hu MY, Zhang YB, Zeng Y (2011b) Biodegradation of deltamethrin and its hydrolysis product 3-phenoxybenzaldehyde by a newly isolated Streptomyces aureus strain HP-S-01. Appl Microbiol Biotechnol 90(4):1471-1483

Chen SH, Yang L, Hu MY, Liu JJ (2011c) Biodegradation of fenvalerate and 3-phenoxybenzoic acid by a novel Stenotrophomonas sp. strain ZS-S-01 and its use in bioremediation of contaminated soils. Appl Microbiol Biotechnol 90(2):755-767

Chen SH, Hu W, Xiao Y, Deng YY, Jia JW, Hu MY (2012a) Degradation of 3-Phenoxybenzoic acid by a Bacillus sp. PLoS ONE 7:e5045611

Chen SH, Luo JJ, Hu MY, Lai KP, Geng P, Huang HS (2012b) Enhancement of cypermethrin degradation by a coculture of Bacillus cereus $\mathrm{ZH}-3$ and Streptomyces aureus HP-S-01. Bioresour Technol 110:97-104

Chen SH, Deng YY, Chang CQ, Lee J, Cheng YY, Cui ZN, Zhou JA, He F, Hu MY, Zhang LH (2015) Pathway and kinetics of cyhalothrin biodegradation by Bacillus thuringiensis strain ZS-19. Sci Rep 5:8784

Cycoń M, Piotrowska-Seget Z (2016) Pyrethroid-degrading microorganisms and their potential for the bioremediation of contaminated soils: a review. Front Microbiol 7:1463

Deng WQ, Liu DR, Yao K, Yuan HY, Wang ZL, Li JL, Zou LK, Han XF, Zhou K, He L, Hu XJ (2015) Liu SL (2015) Characterization of a novel beta-cypermethrin-degrading Aspergillus niger YAT strain and the biochemical degradation pathway of $\beta$-cypermethrin. Appl Microbiol Biotechnol 99(19):8187-8198

Halden RU, Tepp SM, Halden BG, Dwyer DF (1999) Degradation of 3-phenoxybenzoic acid in soil by Pseudomonas pseudoalcaligenes POB310 (pPOB) and two modified Pseudomonas strains. Appl Environ Microbiol 65(8):3354-3359

Hao XX, Zhang XQ, Duan BH, Huo SS, Lin WZ, Xia XX, Liu K (2018) Screening and genome sequencing of deltamethrin-degrading bacterium ZJ6. Curr Microbiol 75(11):1468-1476

Hu KD, Deng WQ, Zhu YT, Yao K, Li JY, Liu AP, Ao XL, Zou LK, Zhou k, He L, Chen SJ, Yang Y, Liu SL (2018) Simultaneous degradation of beta-cypermethrin and 3-phenoxybenzoic acid by Eurotium cristatum ET1, a novel "golden flower fungus" strain isolated from Fu Brick Tea. Microbiologyopen e776

Jones JA, Wang X (2018) Use of bacterial co-cultures for the efficient production of chemicals. Curr Opin Biotechnol 53:33-38

Kamyabi A, Nouri H, Moghimi H (2018) Characterization of pyrene degradation and metabolite identification by Basidioascus persicus and mineralization enhancement with bacterial-yeast co-culture. Ecotoxicol Environ Safe $163: 471-477$

Kuppusamy S, Thavamani P, Megharaj M, Lee YB, Naidu R (2016) Kinetics of PAH degradation by a new acid-metal-tolerant Trabulsiella isolated from the MGP site soil and identification of its potential to fix nitrogen and solubilize phosphorous. J Hazard Mater 307:99-107

Li QY, Li Y, Zhu XK, Cai BL (2008) Isolation and characterization of atrazinedegrading Arthrobacter sp. AD26 and use of this strain in bioremediation of contaminated soil. J Environ Sci 20(10):1226-1230

Liu FF, Chi YL, Wu S, Jia DY, Yao K (2014) Simultaneous degradation of cypermethrin and its metabolite, 3-phenoxybenzoic Acid, by the cooperation of Bacillus licheniformis B-1 and Sphingomonas sp. SC-1. J Agr Food Chem 62(33):8256-8262

Liu J, Huang WW, Han HT, She CC, Zhong GH (2015) Characterization of cellfree extracts from fenpropathrin-degrading strain Bacillus cereus ZH-3 and its potential for bioremediation of pyrethroid-contaminated soils. Sci Total Environ 523:50-58

Tang J, Yao K, Liu SL, Jia DY, Chi YL, Zeng CY, Wu S (2013) Biodegradation of 3-phenoxybenzoic acid by a novel Sphingonas sp. SC-1. Fresenius Environ Bull 22(5A):1564-1572

Tang J, Liu B, Chen TT, Yao K, Zeng L, Zeng CY, Zhang Q (2018a) Screening of a beta-cypermethrin-degrading bacterial strain Brevibacillus parabrevis BCP-09 and its biochemical degradation pathway. Biodegradation 29(6):525-541

Tang J, Liu B, Shi Y, Zeng CY, Chen TT, Zeng L, Zhang Q (2018b) Isolation, identification, and fenvalerate-degrading potential of Bacillus licheniformis CY-012. Biotechnol Biotechnol Equip 32(3):574-582 
Tang J, Hu Q, Liu B, Lei D, Chen TT, Sun Q, Zeng CY, Zhang Q (2019) Efficient biodegradation of 3-phenoxybenzoic acid and pyrethroid pesticides by the novel strain Klebsiella pneumoniae BPBA052. Can J Microbiol. 65(11):795-804

Topp E, Akhtar MH (1991) Identification and characterization of a pseudomonas strain capable of metabolizing phenoxybenzoates. Appl Environ Microbiol 57(5):1294-1300

Tran NH, Urase T, Ngo HH, Hu JY, Ong SL (2013) Insight into metabolic and cometabolic activities of autotrophic and heterotrophic microorganisms in the biodegradation of emerging trace organic contaminants. Bioresour Technol 146:721-731

Yang JJ, Feng YM, Zhan H, Liu J, Yang F, Zhang KY, Zhang LH, Chen SH (2018) Characterization of a pyrethroid-degrading Pseudomonas fulva Strain P31 and biochemical degradation pathway of D-phenothrin. Front Microbiol 9:1003

Zhan H, Wang HS, Liao LS, Feng YM, Fan XH, Zhang LH, Chen SH (2018) Kinetics and novel degradation pathway of permethrin in Acinetobacter baumannii ZH-14. Front Microbiol 9:98

Zhang C, Jia L, Wang SH, Qu J, Li K, Xu LL, Shi YH, Yan YC (2010) Biodegradation of beta-cypermethrin by two Serratia spp. with different cell surface hydrophobicity. Bioresour Technol 101(10):3423-3429
Zhang H, Zhang YM, Hou ZG, Wang XM, Wang J, Lu ZB, Zhao XF, Sun FJ, Pan HY (2016) Biodegradation potential of deltamethrin by the Bacillus cereus strain $Y 1$ in both culture and contaminated soil. Int Biodeterior Biodegrad 106:53-59

Zhao JY, Chi YL, Xu YC, Jia DY, Yao K (2016) Co-Metabolic degradation of beta-cypermethrin and 3-phenoxybenzoic acid by co-Culture of Bacillus licheniformis B-1 and Aspergillus oryzae M-4. PloS One 11:e016679611

Zhu YT, Li JL, Yao K, Zhao N, Zhou K, Hu XJ, Zou LK, Han XF, Liu AP, Liu SL (2016) Degradation of 3-phenoxybenzoic acid by a filamentous fungus AspergilIus oryzae M-4 strain with self-protection transformation. Appl Microbiol Biotechnol 100(22):9773-9786

\section{Publisher's Note}

Springer Nature remains neutral with regard to jurisdictional claims in published maps and institutional affiliations.

\section{Submit your manuscript to a SpringerOpen ${ }^{\odot}$ journal and benefit from:}

- Convenient online submission

- Rigorous peer review

- Open access: articles freely available online

- High visibility within the field

- Retaining the copyright to your article

Submit your next manuscript at $\boldsymbol{\nabla}$ springeropen.com 